\title{
Evidenzbasierte Stimmtherapieprogramme bei Dysphonien: eine Literaturstudie
}

\section{Evidenced-Based Voice Therapy Programs for the Treatment of Dysphonia: a Systematic Literature Review}

Ben Barsties v. Latoszek

\begin{abstract}
Es wurde eine systematische Literaturstudie über evidenzbasierte Stimmtherapieprogramme bei der Behandlung von Dysphonien durchgeführt. 9 von 17 Stimmtherapieprogrammen entsprachen den Selektionskriterien und diese zeigten signifikante Verbesserung von laryngealer Beurteilung, Stimmqualität, Stimmfunktion und/oder Selbstwahrnehmung bei Patienten mit Dysphonien in einem Zeitraum von einem Tag bis 10 Wochen. Die meisten dieser Programme haben einen physiologischen Ansatz.
\end{abstract}

\section{LERNZIEL}

Dieser Artikel soll der Leserin bzw. dem Leser einen Überblick über evidenzbasierte Stimmtherapieprogramme geben, mit denen Stimmstörungen effektiv behandelt werden können.

\section{Einleitung}

Die Prävalenz von Stimmstörungen wird auf bis zu 15\% der erwachsenen Bevölkerung geschätzt und einer von 13 Erwachsenen leidet jährlich unter Stimmproblemen [1]. In bestimmten Bevölkerungsgruppen sind die Werte jedoch erheblich höher, wie z. B. bei Frauen (ca. doppelt so häufig betroffen wie Männer) [1-4], in Stadtgebieten (85\% Prävalenz) [2] und bei Berufssprechern (71,9\% Prävalenz) [3].

Verschiedene Literaturstudien haben gezeigt, dass die Stimmtherapie eine wirksame Behandlungsmethode bei Patienten mit verschiedenen Arten von Dysphonien ist [5-8]. In der Stimmtherapie wird zwischen indirekter und direkter Therapie unterschieden:

- Indirekte Stimmtherapie: Diese ist meist der Beginn von vielen klinischen Interventionen bei der Behandlung von Dysphonien und beschreibt eine individuell für den einzelnen Stimmpatienten angepasste Form von Stimmhygiene. Unzureichende Stimmhygiene kann nämlich zur Entwicklung von erheblichen Stimmproblemen beitragen. Beispiele für unzureichende Stimmhygiene sind Schreien, über den Lärm hinweg Sprechen, häufiges Husten oder Räuspern und geringe Hydration [9].

- Direkte Stimmtherapie: Diese Verfahren umfassen die symptomatische, die physiologische und die eklektische Stimmtherapie [9]:

- Die symptomatische Stimmtherapie bezieht sich auf die Modifikation verschiedener stimmlicher Auffälligkeiten von Stimmsymptomen wie z. B. erhöhter Stimmtonlage, zu leiser oder zu lauter Stimme, erhöhtem laryngealem Muskeltonus usw.

- Die physiologische Stimmtherapie beinhaltet die direkte Modifikation des physiologischen Ablaufs des Stimmmechanismus. In einem normalen Stimmproduktionsablauf herrscht eine ausgewogene Balance zwischen Luftstrom (respiratorischem System), laryngealem Muskeltonus (Balance, Koordination und Ausdauer) und supraglottalen resonatorischen Strukturen (Pharynx, oralem und nasalem Raum). Jegliche Störung dieser physiologischen Balance der Sprechsubsysteme dürfte auch zu Stimmabweichungen führen.

- Die eklektische Form der Stimmtherapie ist eine Kombination aus allen genannten Orientierungen in der Stimmtherapie.

Merke

Eine eigenständige Form stellt die psychogene Stimmtherapie dar, die sich auf die Identifikation und Modifikation von emotionalen und psychosozialen Störungen konzentriert, die mit dem Beginn der Stimmprobleme assoziiert werden [9]. 
Die in diesem Beitrag vorgestellte Studie ist eine Literaturstudie über direkte Stimmtherapieprogramme, die bei Stimmpatienten evaluiert und in Peer-Review-Fachzeitschriften veröffentlicht wurden. Jedes Stimmtherapieprogramm muss einen konzeptionellen Aufbau mit einer Hierarchie aufweisen.

\section{Methode}

Zuerst wurde eine manuelle Suche in der deutsch- und englischsprachigen Literatur durchgeführt, um geeignete Stimmtherapieprogramme herauszufiltern, die bei Patienten mit unterschiedlichen Arten von Dysphonien anzuwenden sind und eine direkte Stimmtherapie implizieren. Im Fokus stehen Stimmtherapieprogramme, die einen symptomatischen, physiologischen oder eklektischen Ansatz haben. Ausgeschlossen wurden auch jegliche Programme mit einem Schwerpunkt im Bereich Prävention. Dazu wurde in geeigneter Fachliteratur gesucht wie z.B. in Fachzeitschriften und Fachbüchern. Die Suche ergab folgendes Ergebnis von potenziellen Stimmtherapieprogrammen für die weitere systematische Literaturstudie:

- Akzentmethode,

- Circumlaryngeal manual Therapy,

- Comprehensive Voice Rehabilitation Program,

- Laryngeal manual Therapy,

- Lessac-Madsen resonant Voice Therapy,

- Novafon local Vibration Voice Therapy,

- Stemple's Resonance Therapy,

- Stretch and Flow Phonation,

- Vocal Function Exercises,

- Larynxmanipulation,

- manuelle Stimmtherapie,

- Nasalierungsmethode,

- atemrhythmisch angepasste Phonation,

- tonale Stimmtherapie,

- Schlaffhorst-Andersen-Methode,

- integrative Stimmtherapie,

- funktionale Stimmtherapie.

Für die weitere Analyse der ausgewählten Stimmtherapieprogramme wurde eine systematische Literaturstudie in verschiedenen Datenbanken (Cochrane Library, Google Scholar und PubMed) durchgeführt. Es wurden dabei nur Studien in englischer oder deutscher Sprache berücksichtigt, die bis zum Jahre 2018 einem Peer-Review-Verfahren unterzogen worden waren. Ebenfalls wurden nur Stimmtherapieprogramme inkludiert, die ein Prä-Post-Design und eine Signifikanz bestimmt hatten, um die Effektivität des jeweiligen Programms nachzuweisen. Ausgeschlossen wurden Studien, die ausschließlich an Probanden mit Dysarthrien oder Dysphagien oder an Stimmgesunden durchgeführt worden waren. Auch wurden keine Studien berücksichtigt, die das Stimmtherapieprogramm als Präventionsmittel oder die mehrere Stimmtherapien mit und ohne medi- zinische Eingriffe für eine Interventionsgruppe genutzt hatten. Gesucht wurde nach dem jeweiligen Namen des Stimmtherapieprogramms und nach den Begriffen „Stimmtherapie“ bzw. „Voice Therapy“.

\section{Ergebnisse}

Das in > Abb. 1 wiedergegebene Ablaufdiagramm zeigt das Selektionsergebnis der durchgeführten systematischen Literaturstudie: Insgesamt wurden 9 von 17 Stimmtherapieprogrammen in den verschiedenen Datenbanken gefunden, die in Peer-Review-Fachzeitschriften an Dysphoniepatienten evaluiert worden waren. Zwei der 9 Stimmtherapiemethoden haben eine symptomatische Stimmtherapie als Schwerpunkt (Circumlaryngeal manual Therapy und Laryngeal manual Therapy) und folgen beide einem manuellen Ansatz, um den laryngealen Muskeltonus zu regulieren. Weitere 5 Stimmtherapiemethoden haben einen physiologischen Ansatz (Vocal Function Exercises, Akzentmethode, Stretch and Flow Phonation, Lessac-Madsen resonant Voice Therapy und Stemple's Resonance Therapy). Die 2 letzten ermittelten Stimmtherapieprogramme wenden ein eklektisches Verfahren an (Novafon lo-

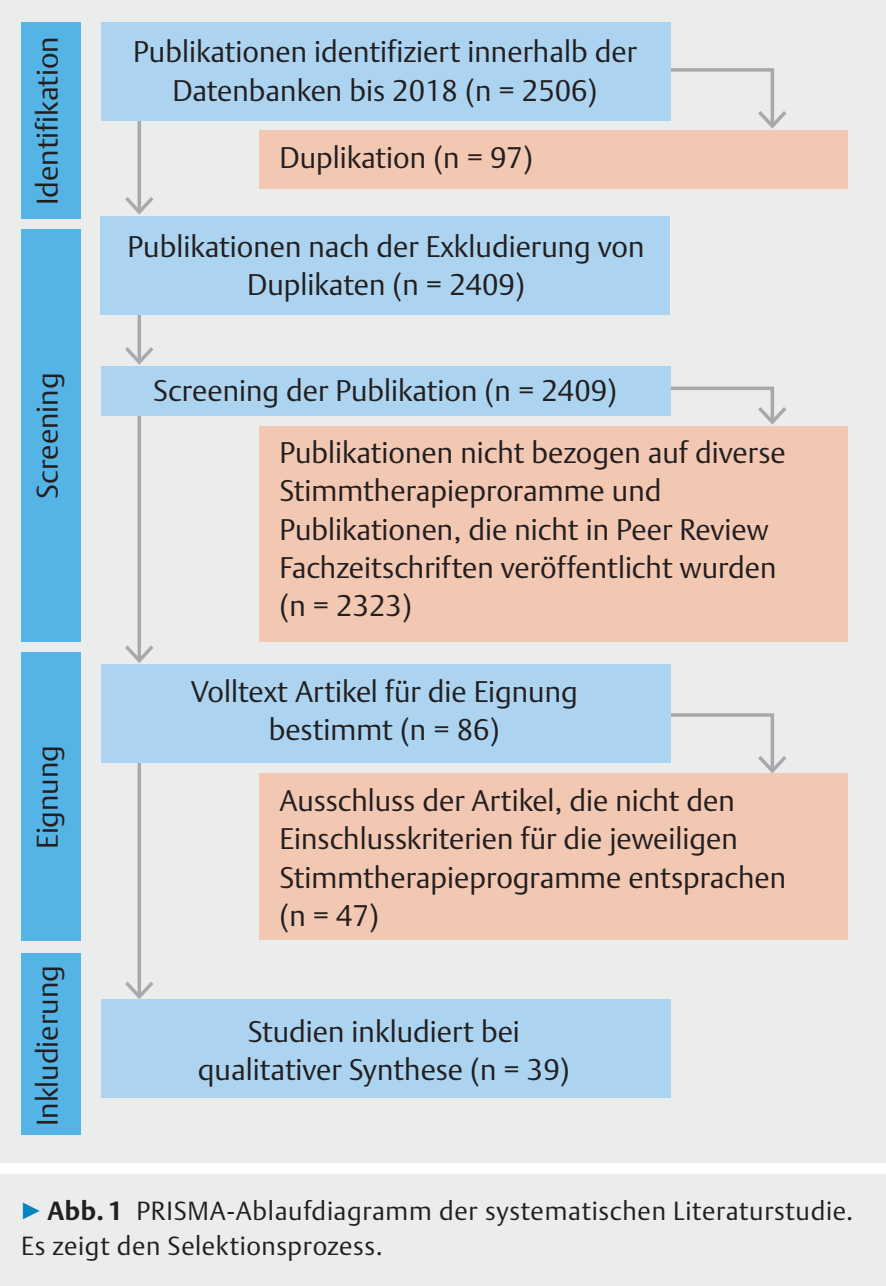


cal Vibration Voice Therapy und Comprehensive Voice Rehabilitation Program).

Der folgende Abschnitt listet die Ergebnisse auf, die durch den Einsatz des jeweiligen Stimmtherapieprogramms zu erwarten sind, sortiert nach der Häufigkeit der Studien zu jedem Stimmprogramm.

\section{Vocal Function Exercises}

Dieses Therapieprogramm wurde in 13 verschiedenen Studien evaluiert [10-22]. Die Therapie erfolgte bei diversen Stimmpathologien über 4-12 Wochen. Bei dieser Methode werden lediglich 4 Übungen durchgeführt: eine Aufwärmübung auf einem nasalen /i/-Laut und 3 weitere Übungen (Stretching, Kontraktion und Kraftübung) auf einem modifizierten /o/-Laut durch die Lippen, sodass ein Lippensummen entsteht. Die Ergebnisse zeigten signifikante Verbesserungen in der laryngealen Beurteilung [18, 19,21], in der perzeptiven und/oder objektiven Stimmqualität [13-15, 18, 19, 21, $22]$, in der Tonhöhe $[13,16]$, in der Aerodynamik [12, $15,18]$ und in der Selbstwahrnehmung $[11,13,14,17-$ 22].

\section{Circumlaryngeal manual Therapy}

Die Circumlaryngeal manual Therapy ist in 10 Studien bei Patienten mit funktioneller Dysphonie und Mutationsfalsettstimmen getestet worden [23-32]. Es ist eine manuelle Methode, um eine muskuläre laryngeale Anspannung zu reduzieren, die mit einer stimmlichen Hyperfunktion assoziiert wird. Dabei werden ausschließlich die laryngealen Strukturen und das Os hyoideum sowie in besonderen Fällen weitere mediale und laterale suprahyoidale Strukturen behandelt. Die Methode wird in der Regel in einer Behandlung evaluiert, da danach bereits signifikante Verbesserungen eintreten können. Die Ergebnisse zeigten in allen Studien signifikante Verbesserungen in objektiver und subjektiver Stimmqualität, die selbst über 3:6, 6:0 und 16:5 Monate anhalten können [25,32]. Auch die Stimmfunktion verbesserte sich signifikant nach der Behandlung [30]. Ebenfalls konnten eine Absenkung der Formanten und artikulatorische Verbesserungen nachgewiesen werden $[26,28,29]$. Jedoch blieb die Tonhöhe in der Regel unverändert, konnte in Einzelfällen aber auch um 6 Halbtöne fallen oder um 16,7 Halbtöne steigen [24, 27]. Die Methode sorgt aber auch für eine erfolgreiche Behandlung bei Mutationsfalsettstimmen bzgl. Tonhöhe und objektiver Stimmqualität [31].

\section{Akzentmethode}

Diese Behandlungsmethode wurde in 4 Studien evaluiert [33-36]. Die Behandlung wurde bei diversen Stimmpathologien angewandt und erstreckte sich über einen individuellen Zeitraum von zwischen 5 und in der Regel 10 Wochen. Das Programm ist systematisch aufgebaut und inkludiert einzelne Bereiche von
Respiration, Phonation, Artikulation und Körperbewegung. Die Ergebnisse nach der Behandlung zeigten signifikante Verbesserungen in laryngealer Beurteilung $[33,35]$, perzeptiver und/oder objektiver Stimmqualität [33-36], Aerodynamik [33,35], Intensität [35,36] und Selbstwahrnehmung [34-36].

\section{Laryngeal manual Therapy}

Die Laryngeal manual Therapy wurde in 4 Studien evaluiert und bei diversen Stimmpathologien getestet [37-40]. Diese manuelle Therapie soll eine Reduktion des Muskeltonus rund um den Larynx bzgl. des M. sternocleidomastoideus, des supralaryngealen Gebiets, des Os hyoideum und des Larynx selbst hervorrufen. Auch bei dieser Therapiemethode kann schon nach einer Behandlung ein Effekt eintreten, der nach einer Woche weiterhin signifikant anhält [37]. Insbesondere wurden Symptome wie Schmerzen in verschiedenen Bereichen, Engegefühl, Trockenheit, Kitzeln, Wundgefühl und Gereiztheit geringer [37-40]. Die objektiven oder perzeptiven Stimmqualitätsparameter verbesserten sich nach der Behandlung in den meisten Studien dagegen nicht signifikant [37-39] und die Tonhöhe wies auch keine signifikante Veränderung auf [37].

\section{Novafon local Vibration Voice Therapy}

Die Literatursuche nach Novafon local Vibration Voice Therapy lieferte ein Ergebnis von 2 Studien [41,42]. Bei dieser Therapie wird der Patient über 5 Wochen mit Stimmübungen (diversen Semi-occluded-vocalTract- und Resonanzübungen) und dem Novafongerät (Novafon classic; Novafon GmbH, Weinstadt), einem lokalen Vibrationsgerät, behandelt. Die Probanden in beiden Studien hatten diverse organische und funktionelle Dysphonien. Generell zeigten beide Studien, dass die Heiserkeit, das Stimmumfangsprofil und die Selbstwahrnehmung der Stimmstörung sich signifikant verbesserten. Der aerodynamische Aspekt, gemessen mit dem Phonationsquotienten, erreichte keine signifikante Verbesserung nach der Therapie im Vergleich zum Zustand vorher. Allerdings zeigte sich, dass bereits nach 3 Wochen im Bereich der Stimmqualität signifikante Verbesserungen eintreten können [41]. Der Mehrwert der Anwendung von lokaler Vibrationstherapie zusätzlich zu Stimmübungen konnte aktuell nur im Bereich der Stimmqualität eindeutig belegt werden [42].

\section{Stretch and Flow Phonation}

Die Stretch and Flow Phonation wurde ebenfalls in 2 Studien getestet $[43,44]$. Dabei wurden nur Patienten mit funktioneller Dysphonie inkludiert und 6 Wochen lang behandelt. Die Patienten durchlaufen bei dieser Behandlungsmethode 5 Stufen, in denen sie von der Kontrolle über den stimmlosen unforcierten Luftstrom über behauchte Abstufungen von Stimmgebung und Tempowechsel bis zur klaren Stimmqualität ohne An- 
spannung gelangen. Nach der Behandlung waren in beiden Studien signifikante Verbesserungen im Bereich der objektiven Stimmqualität, der Aerodynamik und der Selbstwahrnehmung erreicht worden $[43,44]$.

\section{Comprehensive Voice Rehabilitation Program}

Dieses Therapieprogramm wurde auch in 2 Studien evaluiert; es wurden dabei Patienten mit einer funktionellen Dysphonie untersucht $[21,45]$. Es handelt sich um ein Programm, das die Wahrnehmung schult, Bewusstsein bzw. Aufklärung ermöglicht und negative Gewohnheiten verändert. Darüber hinaus wird an der Körperhaltung, der Stimmfähigkeit, Resonanzübungen, der Atmungskontrolle, der Artikulation und der Stimmprojektion gearbeitet. Der Behandlungszeitraum dieses Programms erstreckt sich über 6 Wochen. Nach der Behandlung zeigten sich signifikante Verbesserungen in unterschiedlichen Aspekten der Selbstwahrnehmung der Stimmstörung, in der laryngealen Beurteilung und bei der perzeptiven sowie der objektiven Stimmqualität.

\section{Lessac-Madsen resonant Voice Therapy}

Die Lessac-Madsen resonant Voice Therapy wurde in einer Studie bei weiblichen Lehrern mit Stimmstörungen in einem Behandlungszeitraum von 8 Wochen getestet. Das Therapieprogramm gliedert sich in 8 Stufen, um eine resonanzvolle Stimme aufzubauen und als Zielstimme zu verwenden [46]. Nach der Behandlung zeigten sich signifikante Verbesserungen in unterschiedlichen Aspekten der laryngealen Beurteilung, der physischen Selbstwahrnehmung der Stimmstörung, der Tonhöhe, der Sprechmelodie und der Dynamik, in wenigen Bereichen der Aerodynamik und in der perzeptiven Stimmqualität. Jedoch wurden keine signifikanten Unterschiede in der objektiven Stimmqualität festgestellt.

\section{Stemple's Resonance Therapy}

Die Stemple's Resonance Therapy wurde auch in einer Studie bei Lehrern mit funktionellen Stimmstörungen überprüft [47]. Die Probandengruppe wurde 6 Wochen lang therapiert. Die Therapie gliedert sich in 9 Stufen mit dem Ziel, eine resonanzvolle Stimme aufzubauen und als Zielstimme zu verwenden. Nach der Stemple's Resonance Therapy wurden signifikante Verbesserungen in der Selbstwahrnehmung der Stimmstörung gemessen.

\section{Diskussion}

Das Ziel der beschriebenen Studie war, einen Überblick über die Vielzahl von Stimmtherapiemethoden zu geben, die in der Literatur auf ihre Effektivität überprüft worden sind. Dabei war es wichtig aufzulisten, in welchen Bereichen der Stimme und über welchen Zeitraum Verbesserungen eintreten können. Es zeigte sich, dass alle Programme eine Verbesserung in der Multidimensionalität der Stimme erreichen können. Diese Verbesserung kann je nach Behandlungsart und Störungsbild bereits nach einer Behandlung oder in den meisten Fällen nach 3-10 Wochen eintreten.

\section{Merke}

Somit sind alle diese Programme für die Behandlung von Dysphonien zu empfehlen; ihre Eignung ist dabei für jeden Patienten je nach individuellem Schwerpunkt und Bedürfnis sowie nach individueller Verträglichkeit und Durchführbarkeit abzuwägen.

Hinsichtlich der Vergleichbarkeit der Ergebnisse der oben aufgelisteten Programme ist nur eine Studie bekannt, die Programme miteinander verglichen hat [21]: Das ganzheitliche und umfangreiche Comprehensive Voice Rehabilitation Program wurde mit den Vocal Function Exercises verglichen. Das gestraffte VocalFunction-Exercises-Programm mit 4 Übungen erreichte statistisch vergleichbare Resultate wie das Comprehensive Voice Rehabilitation Program. Weitere Vergleiche der aufgelisteten Programme mit anderen Methoden bzw. Anwendungen (z. B. Phonation Resistance Training [17], Stimmverstärker im Unterricht $[19,47]$, FlowResistant Tube [20], Atmungstraining/Therapie [30, 47], eklektische Methoden [36], transkutane elektrische Nervenstimulanz [38, 40] und Stimmhygiene [44]) oder mit einer Kontrollgruppe ohne Therapie [11,1520] wurden bei 6 von den 9 Programmen durchgeführt. Dabei fielen die Ergebnisse unterschiedlich aus.

Es gibt eine Anzahl von Einschränkungen, die die Generalisierbarkeit der Erkenntnisse dieser systematischen Literaturstudie begrenzen, aber sie gibt eine Richtung für zukünftige Arbeiten vor:

- Nur 4 Stimmtherapieprogramme (Akzentmethode, Circumlaryngeal manual Therapy, Laryngeal manual Therapy und Vocal Function Exercises) wurden mehrfach auf ihre Effektivität überprüft. Somit sind die Diversität von damit behandelten Stimmstörungsbildern und die Reproduzierbarkeit der Ergebnisse bei den meisten Programmen noch ausbaufähig.

- Es wurden nur Stimmtherapieprogramme mit direktem Stimmtherapieansatz berücksichtigt. Die Stimmtherapie ist jedoch noch vielfältiger und umfangreicher als in der Literaturstudie geschildert. Ausgeschlossen wurden z. B. reine psychogene Stimmtherapieansätze, alleinige Stimmübungen (z. B. Summen [48, 49], einzelne Semi-occludedvocal-Tract-Übungen [50]), Präventionsprogramme und indirekte Stimmtherapieansätze.

- Es wurde keine Analyse zum Grad der Evidenzen der einzelnen Studien durchgeführt. Dies wäre aber wichtig, um die Qualität der Studien zu bestimmen. Einfluss darauf haben Faktoren, die die Qualität 
einer Studie herabsetzen, wie z. B. Studieneinschränkung, mögliche Risiken oder Bias, Anspielung von Evidenz, Diskrepanzen zwischen Studien ohne plausible Erklärung, Ungenauigkeiten der Resultate und Verdacht auf Publikationsbias. Hingegen wird die Qualität einer Studie aufgewertet durch Faktoren wie z.B. den Dosiswirkungsgradienten, einen starken Anstieg von Effekten und Bias in anderen Studien, der den demonstrierten Effekt unterschätzt. All diese Faktoren gelten als nötig für die Evidenzgradermittlung.

- Es wurde in den Studien keine Metaanalyse der gewonnenen Daten durchgeführt, um diese Studienresultate systematisch zu kombinieren und zu bewerten.

- Es wurden nur deutsch- und englischsprachige Studien in der Literaturstudie berücksichtigt. Es gibt jedoch zahlreiche relevante Zeitschriften und Studien, die in anderen Sprachen veröffentlicht wurden.

\section{FAZIT}

Die Literaturstudie zeigte, dass 9 direkte Stimmtherapieprogramme eine signifikante Verbesserung im Bereich laryngealer Beurteilung, Stimmqualität, Stimmfunktion und Selbstwahrnehmung bei Patienten mit Dysphonien in einem Zeitraum von einem Tag bis 10 Wochen erzielen lassen. Die meisten dieser Programme haben einen physiologischen Ansatz.

\section{Schlüsselwörter}

Stimmtherapie, Dysphonie, Therapieeffekte, systematische Literaturstudie, Therapieresultate

\section{Interessenkonflikt}

Die Autorinnen/Autoren geben an, dass kein interessenkonflikt besteht

\section{Autorinnen/Autoren}

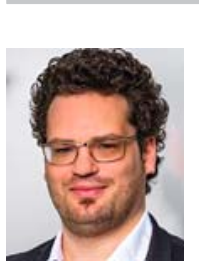

\section{Ben Barsties v. Latoszek ${ }^{1,2}$}

Prof. Dr.; Professor zur Vertretung für Logopädie, Schwerpunkt Stimme, an der SRH Hochschule für Gesundheit in Düsseldorf, dort Betreuung des Studiengangs Logopädie, ausbildungsintegrierend, mit langjähriger Erfahrung in der Diagnostik und Therapie bei

Stimmstörungen. Klinische Tätigkeit als Stimmtherapeut im Therapiezentrum Dormagen.

\section{Institute}

1 Logopädie, SRH Hochschule für Gesundheit, Düsseldorf

2 Stimmtherapie, Therapiezentrum Dormagen, Dormagen

\section{Korrespondenzadresse}

Prof. Dr. Ben Barsties v. Latoszek

Logopädie

SRH Hochschule für Gesundheit, Düsseldorf

Graf-Adolf-Straße 67

40210 Düsseldorf

E-Mail: benjamin.latoszek@srh.de

\section{Literatur}

[1] Bhattacharyya N. The prevalence of voice problems among adults in the United States. Laryngoscope 2014; 124: 23592362

[2] Cohen SM, Kim J, Roy N et al. Prevalence and causes of dysphonia in a large treatment-seeking population. Laryngoscope 2012; 122: 343-348

[3] Mozzanica F, Ginocchio D, Barillari R et al. Prevalence and voice characteristics of laryngeal pathology in an Italian voice therapy-seeking population. J Voice 2016; 30: 774. e13-774.e21

[4] De Bodt M, Van den Steen L, Mertens F et al. Characteristics of a dysphonic population referred for voice assessment and/or voice therapy. Folia Phoniatr Logop 2015; 67: 178186

[5] Ruotsalainen J, Sellman J, Lehto L et al. Systematic review of the treatment of functional dysphonia and prevention of voice disorders. Otolaryngol Head Neck Surg 2008; 138: 557-565

[6] Speyer R. Effects of voice therapy: a systematic review. J Voice 2008; 22: 565-580

[7] Desjardins M, Halstead L, Cooke M et al. A systematic review of voice therapy: what „effectiveness" really implies. J Voice 2017; 31: 392.e13-392.e32

[8] Da Cunha Pereira G, de Oliveira Lemos I, Dalbosco Gadenz C et al. Effects of voice therapy on muscle tension dysphonia: a systematic literature Review. J Voice 2018; 32: 546-552

[9] Stemple JC, Roy N, Klaben BK. Clinical voice pathology: theory and managment. 5th ed. San Diego: Plural Publishing; 2014

[10] Roy N, Gray SD, Simon M et al. An evaluation of the effects of two treatment approaches for teachers with voice disorders: a prospective randomized clinical trial. J Speech Lang Hear Res 2001; 44: 286-296

[11] Gillivan-Murphy P, Drinnan M], O'Dwyer TP et al. The effectiveness of a voice treatment approach for teachers with self-reported voice problems. J Voice 2006; 20: 423-431

[12] Gorman S, Weinrich B, Lee L et al. Aerodynamic changes as a result of vocal function exercises in elderly men. Laryngoscope 2008; 118: 1900-1903

[13] Nguyen DD, Kenny DT. Randomized controlled trial of vocal function exercises on muscle tension dysphonia in Vietna- 
mese female teachers. J Otolaryngol Head Neck Surg 2009; 38: $261-278$

[14] Sauder C, Roy N, Tanner K et al. Vocal function exercises for presbylaryngis: a multidimensional assessment of treatment outcomes. Ann Otol Rhinol Laryngol 2010; 119: 460467

[15] Tay EY, Phyland DJ, Oates ]. The effect of vocal function exercises on the voices of aging community choral singers. J Voice 2012; 26: 672.e19-672.e27

[16] Gelfer MP, Van Dong BR. A preliminary study on the use of vocal function exercises to improve voice in male-to-female transgender clients. J Voice 2013; 27: 321-334

[17] Ziegler A, Verdolini AbbottK, Johns M et al. Preliminary data on two voice therapy interventions in the treatment of presbyphonia. Laryngoscope 2014; 124: 1869-1876

[18] Kaneko M, Hirano S, Tateya I et al. Multidimensional analysis on the effect of vocal function exercises on aged vocal fold atrophy. J Voice 2015; 29: 638-644

[19] Teixeira LC, Behlau M. Comparison between vocal function exercises and voice amplification. J Voice 2015; 29: 718726

[20] Kapsner-Smith MR, Hunter E], Kirkham K et al. A randomized controlled trial of two semi-occluded vocal tract voice therapy protocols. J Speech Lang Hear Res 2015; 58: 535-549

[21] Pedrosa V, Pontes A, Pontes P et al. The effectiveness of the comprehensive voice rehabilitation program compared with the vocal function exercises method in behavioral dysphonia: a randomized clinical trial. J Voice 2016; 30: 377.e11377.e19

[22] Jafari N, Salehi A, Izadi F et al. Vocal function exercises for muscle tension dysphonia: auditory-perceptual evaluation and self-assessment rating. J Voice 2017; 31: 506.e25-506. e31

[23] Roy N, Leeper HA. Effects of the manual laryngeal musculoskeletal tension reduction technique as a treatment for functional voice disorders: perceptual and acoustic measures. J Voice 1993; 7: 242-249

[24] Roy N, Tasko SM. Speaking Fundamental Frequency (SFF) changes following successful management of functional dysphonia. J Speech Language Pathol Audiol 1994; 18: 115120

[25] Roy N, Bless DM, Heisey D et al. Manual circumlaryngeal therapy for functional dysphonia: an evaluation of shortand long-term treatment outcomes. J Voice 1997; 11: 321 331

[26] Roy N, Ferguson NA. Formant frequency changes following manual circumlaryngeal therapy for functional dysphonia: evidence of laryngeal lowering. J Med Speech Language Pathol 2001; 9: 169-175

[27] Roy N, Hendarto H. Revisiting the pitch controversy: changes in Speaking Fundamental Frequency (SFF) after management of functional dysphonia. J Voice 2005; 19 ; 582-591

[28] Dromey C, Nissen SL, Roy N et al. Articulatory changes following treatment of muscle tension dysphonia: preliminary acoustic evidence. J Speech Lang Hear Res 2008; 51: 196208

[29] Roy N, Nissen SL, Dromey C et al. Articulatory changes in muscle tension dysphonia: evidence of vowel space expansion following manual circumlaryngeal therapy. J Commun Disord 2009; 42: 124-135

[30] Van Lierde KM, De Bodt M, Dhaeseleer E et al. The treatment of muscle tension dysphonia: a comparison of two treat- ment techniques by means of an objective multiparameter approach. J Voice 2010; 24: 294-301

[31] Roy N, Peterson EA, Pierce JL et al. Manual laryngeal reposturing as a primary approach for mutational falsetto. Laryngoscope 2017; 127: 645-650

[32] Dehqan A, Scherer RC. Positive effects of manual circumlaryngeal therapy in the treatment of Muscle Tension Dysphonia (MTD): long term treatment outcomes. J Voice 2018: [Epub ahead of print] doi: 10.1016/j.jvoice.2018.07.010

[33] Kotby MN, Sady SRE, Basiouny SE et al. Efficacy of the accent method of voice therapy. J Voice 1991; 5: 316-320

[34] Fex B, Fex S, Shiromoto O et al. Acoustic analysis of functional dysphonia: before and after voice therapy (accent method). J Voice 1994; 8: 163-167

[35] Bassiouny S. Efficacy of the accent method of voice therapy. Folia Phoniatr Logop 1998; 50: 146-164

[36] Stier KH. Behandlung von Stimmpatienten mit der Akzentmethode im Vergleich zu nicht methodenorientierter Stimmtherapie. Stimme Sprache Gehör 2011; 35: e68-e76

[37] Mathieson L, Hirani SP, Epstein R et al. Laryngeal manual therapy: a preliminary study to examine its treatment effects in the management of muscle tension dysphonia. J Voice 2009; 23: 353-366

[38] Alves Silverio KC, Brasolotto AG, Thaís Donalonso Siqueira L et al. Effect of application of transcutaneous electrical nerve stimulation and laryngeal manual therapy in dysphonic women: clinical trial. J Voice 2015; 29: 200-208

[39] Reimann AP, Siqueira LT, Rondon AV et al. Immediate effect of laryngeal manual therapy in dysphonic individuals. Codas 2016; 28: 59-65

[40] Conde MCM, Siqueira LTD, Vendramini JE et al. Transcutaneous Electrical Nerve Stimulation (TENS) and Laryngeal Manual Therapy (LMT): immediate effects in women with dysphonia. J Voice 2018; 32 : 385.e17-385.e25

[41] Barsties v Latoszek B. Preliminary study of Novafon local vibration voice therapy for dysphonia treatment. Logoped Phoniatr Vocol 2018: [Epub ahead of print] doi: 10.1080/ 14015439.2018.1453541

[42] Barsties v Latoszek B. Treatment effectiveness of Novafon local vibration voice therapy for dysphonia treatment. J Voice 2018: [Epub ahead of print] doi: 10.1016/j.jvoice. 2018.05.009

[43] Watts C, Diviney S, Hamilton A et al. The effect of stretchand-flow voice therapy on measures of vocal function and handicap. J Voice 2015; 29: 191-199

[44] Watts CR, Hamilton A, Toles L et al. A randomized controlled trial of stretch-and-flow voice therapy for muscle tension dysphonia. Laryngoscope 2015; 125: 1420-1425

[45] Cavalcanti NR, Souza BO, Gama ACC et al. Effect of the comprehensive voice rehabilitation program in teachers with behavioral dysphonia. Codas 2018; 30: e20170182

[46] Chen SH, Hsiao TY, Hsiao LC et al. Outcome of resonant voice therapy for female teachers with voice disorders: perceptual, physiological, acoustic, aerodynamic, and functional measurements. J Voice 2007; 21: 415-425

[47] Roy N, Weinrich B, Gray SD et al. Three treatments for teachers with voice disorders: a randomized clinical trial. J Speech Lang Hear Res 2003; 46: 670-688

[48] Ogawa M, Hosokawa K, Yoshida M et al. Immediate effects of humming on computed electroglottographic parameters in patients with muscle tension dysphonia. J Voice 2014; 28 : $733-741$ 
[49] Vlot C, Ogawa M, Hosokawa K et al. Investigation of the immediate effects of humming on vocal fold vibration irregularity using electroglottography and high-speed laryngoscopy in patients with organic voice disorders. J Voice 2017; 31: 48-56

[50] Andrade PA, Wood G, Ratcliffe P et al. Electroglottographic study of seven semi-occluded exercises: LaxVox, straw, liptrill, tongue-trill, humming, hand-over-mouth, and tonguetrill combined with hand-over-mouth. J Voice 2014; 28 : 589-595

\section{Bibliografie}

DOI https://doi.org/10.1055/a-0949-6520

Online-Publikation: 12.11.2019 | Sprache · Stimme · Gehör 2020; 44: 16-22

(c) Georg Thieme Verlag KG Stuttgart · New York ISSN 0342-0477 